\title{
Existence and Numerical Method for Nonlinear Third-Order Boundary Value Problem in the Reproducing Kernel Space
}

\author{
Xueqin Lï̈ ${ }^{1,2}$ and Minggen Cui ${ }^{1}$ \\ ${ }^{1}$ Department of Mathematics, Harbin Institute of Technology, Weihai, Shandong 264209, China \\ ${ }^{2}$ School of Mathematics and Sciences, Harbin Normal University, Harbin, Heilongjiang 150025, China
}

Correspondence should be addressed to Xueqin Lü, hashidalvxueqin@126.com

Received 14 July 2009; Revised 12 November 2009; Accepted 11 January 2010

Academic Editor: Irena Rachůnkova

Copyright (C) 2010 X. Lü and M. Cui. This is an open access article distributed under the Creative Commons Attribution License, which permits unrestricted use, distribution, and reproduction in any medium, provided the original work is properly cited.

We are concerned with general third-order nonlinear boundary value problems. An existence theorem of solution is given under weaker conditions. In the meantime, an iterative algorithm with global convergence is presented. The higher order derivatives of approximate solution is obtained by using this method can approximate the corresponding derivatives of exact solution well.

\section{Introduction}

The third-order boundary value problems arise in the study of draining and coating flows. In the past few years, third-order boundary value problems with various types of boundary conditions have been studied in many literatures. For details, see [1-6] and their references therein. In the last decade or so, several papers [7-12] have been devoted to the study of third-order differential equations with two point boundary value conditions. In [13], the authors discussed the solvability of a class of particular third-order nonlinear boundary value problems:

$$
\begin{gathered}
u^{(3)}(x)-f\left(x, u(x), u^{\prime}(x), u^{\prime \prime}(x)\right)=0, \quad 0 \leq x \leq 1, \\
u(0)=0, \quad u^{\prime}(0)=0, \quad u^{\prime}(1)=0 .
\end{gathered}
$$

In this paper, we consider third-order nonlinear equations subject to the other boundary value conditions: 


$$
\begin{gathered}
u^{(3)}(x)-f\left(x, u(x), u^{\prime}, u^{\prime \prime}(x)\right)=0, \quad 0 \leq x \leq 1, \\
u(1)=0, \quad u^{\prime}(0)=0, \quad u^{\prime}(1)=0 .
\end{gathered}
$$

In order to derive the existence theorems for solution of (1.2), we make the following assumptions $\mathrm{H}$ :

(H1) $f(x, y, z, w) \in[0,1] \times R^{3}$ is completely continuous function;

(H2) $f(x, y, z, w), f_{x}(x, y, z, w), f_{y}(x, y, z, w), f_{z}(x, y, z, w)$ and $f_{w}(x, y, z, w)$ are bounded;

(H3) $f(x, y, z, w)>0$ on $[0,1] \times R^{3}$,

where $f(x, y, z, w) \in W_{1}[0,1]$ as $y=y(x) \in W_{1}[0,1], z=z(x) \in W_{1}[0,1], w=w(x) \in$ $W_{1}[0,1],(0 \leq x \leq 1,-\infty<y, z, w<\infty)$.

Under weaker conditions, we give an existence theorem. In the meantime, we give an iterative method of obtaining the solution of (1.2). The method has following features: firstly, the method is an iterative method in a wide range, that is, the convergence of iterative sequences is independent of the choice of initial values; secondly, the approximate solution obtained by using this method can approximate the higher order derivatives of exact solution well; thirdly, we consider the problem in the reproducing kernel Hilbert space and use sufficiently the good properties of the space. When we choose an appropriate reproducing kernel Hilbert space, we can discuss higher-order boundary value problems in a similar manner.

\section{Several Reproducing Kernel Spaces}

\subsection{The Reproducing Kernel Space $W_{1}[0,1]$}

The inner product space $W_{1}[0,1]$ is defined by $W_{1}[0,1]=\{u(x) \mid u(x)$ is absolutely continuous real value function in $\left.[0,1], u^{\prime}(x) \in L^{2}[0,1]\right\}$. The inner product and norm in $W_{1}[0,1]$ are given, respectively, by

$$
\langle u(x), v(x)\rangle_{W_{1}}=u(0) v(0)+\int_{0}^{1} u^{\prime}(x) v^{\prime}(x) d x, \quad\|u\|_{W_{1}}=\langle u(x), u(x)\rangle^{1 / 2} .
$$

In $[14,15]$, the author had proved that $W_{1}[0,1]$ is a complete reproducing kernel space. That is, there exists a reproducing kernel function $Q_{x}(y) \in W_{1}[0,1], y \in[0,1]$, for each fixed $x \in[0,1]$ and any $u(y) \in W_{1}[0,1]$, such that $\left\langle u(y), Q_{x}(y)\right\rangle_{W_{1}}=u(x)$. The reproducing kernel $Q_{x}(y)$ can be denoted by

$$
Q_{x}(y)= \begin{cases}1+y, & y \leq x \\ 1+x, & y>x\end{cases}
$$




\subsection{The Reproducing Kernel Space $W_{2}[0,1]$}

The inner product space $W_{2}[0,1]$ is defined by $W_{2}[0,1]=\left\{u(x) \mid u^{(3)}\right.$ are absolutely continuous real value functions, $\left.u^{(4)} \in L^{2}[0,1], u(1)=0, u^{\prime}(0)=0, u^{\prime}(1)=0\right\}$. The inner product and norm in $W_{2}[0,1]$ are given, respectively, by

$$
\langle u(y), v(y)\rangle_{W_{2}}=\sum_{i=0}^{3} u^{(i)}(0) v^{(i)}(0)+\int_{0}^{1} u^{(4)} v^{(4)} d y, \quad\|u\|_{W_{2}}=\sqrt{\langle u, u\rangle_{W_{2}}},
$$

where $u, v \in W_{2}[0,1]$.

Theorem 2.1. The space $W_{2}[0,1]$ is a reproducing kernel space. That is, for any fixed $x \in[0,1]$, there exists $T_{x}(y) \in W_{2}[0,1]$, such that for any $u(x) \in W_{2}[0,1], u(x)=\left\langle u(y), T_{x}(y)\right\rangle_{W_{2}}$. The reproducing kernel $T_{x}(y)$ can be denoted by

$$
T_{x}(y)=\left\{\begin{array}{l}
\frac{1}{11975695200}\left(( - 1 + x ) ^ { 2 } \left(5 0 4 0 \left(17410+34820 x+4350 x^{2}-1340 x^{3}-835 x^{4}\right.\right.\right. \\
+468 x^{5}+1260\left(-191520-383040 x-30110 x^{2}+20200 x^{3}-5145 x^{4}+38 x^{5}\right) y^{2} \\
-140\left(-892080-1784160 x+47340 x^{2}-30970 x^{3}+7300 x^{4}+177 x^{5}\right) y^{3} \\
-35\left(-892080-1784160 x+47340 x^{2}-30970 x^{3}+7300 x^{4}+177 x^{5}\right) y^{4} \\
-21\left(-191520-383040 x-30110 x^{2}+20200 x^{3}-5145 x^{4}+38 x^{5}\right) y^{5} \\
+161\left(-55440-7570 x-560 x^{2}+410 x^{3}-130 x^{4}+11 x^{5}\right) y^{6} \\
\left.\left.-\left(17410+34820 x+4350 x^{2}-1340 x^{3}-835 x^{4}+468 x^{5}\right) y^{7}\right)\right), \quad y \leq x, \\
-\frac{1}{11975695200}\left((-1+y)^{2}(5040\right. \\
-161 x^{6}\left(-55440-7570 y-560 y^{2}+410 y^{3}-130 y^{4}+11 y^{5}\right) \\
-1260 x^{2}\left(-191520-383040 y-30110 y^{2}+20200 y^{3}-5145 y^{4}+38 y^{5}\right)+21 x^{5} \\
\left(-191520-383040 y-30110 y^{2}+20200 y^{3}-5145 y^{4}+38 y^{5}\right)+140 x^{3} \\
\left(-892080-1784160 y+47340 y^{2}-30970 y^{3}+7300 y^{4}+177 y^{5}\right)+35 x^{4} \\
\left(-892080-1784160 y+47340 y^{2}-30970 y^{3}+7300 y^{4}+177 y^{5}\right)-5040 \\
\left(17410+34820 y+4350 y^{2}-1340 y^{3}-835 y^{4}+468 y^{5}\right)+x^{7} \\
\left.\left.\left(17410+34820 y+4350 y^{2}-1340 y^{3}-835 y^{4}+468 y^{5}\right)\right)\right), \quad y>x .
\end{array}\right.
$$

\subsection{The Reproducing Kernel Space $W_{3}[0,1]$}

The inner product space $W_{3}[0,1]$ is defined by $W_{3}[0,1]=\left\{u(x) \mid u^{(3)}\right.$ are absolutely continuous real value functions, $\left.u^{(4)} \in L^{2}[0,1], u(0)=0, u^{\prime}(0)=0, u^{\prime}(1)=0\right\}$. The definitions of inner product and norm in $W_{3}[0,1]$ are the same as $(2.3)$. 
Theorem 2.2. The space $W_{3}[0,1]$ is a reproducing kernel space. That is, for any fixed $x \in[0,1]$, there exists $R_{x}(y) \in W_{3}[0,1]$, such that for any $u(x) \in W_{3}[0,1], u(x)=\left\langle u(y), R_{x}(y)\right\rangle_{W_{3}}$. The reproducing kernel $R_{x}(y)$ can be denoted by

$$
R_{x}(y)=\left\{\begin{array}{l}
-\frac{y^{2}}{4717440}\left(-6552 x y^{4}+936 y^{5}+420 x^{3}\left(360-252 y-63 y^{2}-6 y^{3}+y^{4}\right)\right. \\
+105 x^{4}\left(360+60 y+15 y^{2}-6 y^{3}+y^{4}\right)-42 x^{5}\left(360+60 y+15 y^{2}-6 y^{3}+y^{4}\right) \\
+7 x^{6}\left(360+60 y+15 y^{2}-6 y^{3}+y^{4}\right) \\
\left.+504 x^{2}\left(-540+300 y+75 y^{2}+9 y^{3}+5 y^{4}\right)\right), \quad y \leq x \\
\frac{x^{2}}{4717440}\left(-936 x^{5}+6552 x^{4} y-504\left(-540+300 x+75 x^{2}+9 x^{3}+5 x^{4}\right) y^{2}\right. \\
-420\left(360-252 x-63 x^{2}-6 x^{3}+x^{4}\right) y^{3}-105\left(360+60 x+15 x^{2}-6 x^{3}+x^{4}\right) y^{4} \\
+42\left(360+60 x+15 x^{2}-6 x^{3}+x^{4}\right) y^{5} \\
\left.-7\left(360+60 x+15 x^{2}-6 x^{3}+x^{4}\right) y^{6}\right), \quad y>x .
\end{array}\right.
$$

The method of obtaining the reproducing kernel $T_{x}(y), R_{x}(y)$ and the proofs of Theorem 2.1, Theorem 2.2 are given in [16].

\section{Preliminaries of Constructing Iterative Method}

For the convenience of discussion, let $L: W_{2} \rightarrow W_{1}, L u=u^{(3)}$, then (1.2) can be converted into the form as follows:

$$
L u=f\left(x, u, u^{\prime}, u^{\prime \prime}\right), \quad u \in W_{2}[0,1]
$$

Now, we fix $\widehat{u} \in W_{2}[0,1]$ and denote $f\left(x, \widehat{u}, \widehat{u}^{\prime}, \widehat{u}^{\prime \prime}\right)$ by $f(\widehat{u}) . f(\widehat{u}) \in W_{1}[0,1]$ and $f(\widehat{u})$ is a known function. In order to construct the present iterative method, we will consider the following equation:

$$
L u=f\left(x, \widehat{u}, \widehat{u}^{\prime}, \widehat{u}^{\prime \prime}\right), \quad u \in W_{2}[0,1], f \in W_{1}[0,1] .
$$

We convert (3.1) and (3.2) into the following (3.3) and (3.4), respectively,

$$
\begin{aligned}
L u & =f(u), \\
L u & =f(\widehat{u}) .
\end{aligned}
$$


Now, we construct an orthogonal system of functions firstly.

Let $\varphi_{i}(x)=Q_{x_{i}}(x)$, where $\left\{x_{i}\right\}_{i=1}^{\infty}$ is dense on $[0,1]$. Let $\varphi_{0}=f(\widehat{u})$. The orthogonal system of functions $\left\{\bar{\varphi}_{i}(x)\right\}_{i=0}^{\infty}$ can be derived from Gram-Schmidt orthogonalization process of $\left\{\varphi_{i}(x)\right\}_{i=0}^{\infty}$ :

$$
\bar{\varphi}_{i}(x)=\sum_{k=0}^{i} \alpha_{i k} \varphi_{k}(x)
$$

where $\bar{\varphi}_{0}=\varphi_{0}=f(\widehat{u})$. In order to emphasize the fact that the first function of orthogonal system of functions is $f(\widehat{u})$, we denote $\bar{\varphi}_{i}$ by $\bar{\varphi}_{i}(f(\widehat{u}))$. Put $\psi_{i}(x)=L^{*} \bar{\varphi}_{i}(x) \in W_{2}, i=0,1,2, \ldots$, where $L^{*}$ is the conjugate operator of $L$. Similarly, we denote $\psi_{i}$ by $\psi_{i}(f(\widehat{u}))$.

Now, we introduce some notations:

$\Psi_{n}(f(\widehat{u}))=\operatorname{Span}\left\{\psi_{1}(f(\widehat{u})), \psi_{2}(f(\widehat{u})), \ldots, \psi_{n}(f(\widehat{u}))\right\}$

$\Psi(f(\widehat{u}))=\operatorname{Span}\left\{\psi_{1}(f(\widehat{u})), \psi_{2}(f(\widehat{u})), \ldots\right\} ;$

$P_{n}: W_{2} \rightarrow \Psi_{n}(f(\widehat{u}))$ is a orthogonal projector;

$P: W_{2} \rightarrow \Psi(f(\widehat{u}))$ is a orthogonal projector;

$\Psi_{n}^{\perp}(f(\widehat{u}))$ is the orthogonal complement space of $\Psi_{n}(f(\widehat{u}))$ ；

$\Psi^{\perp}(f(\widehat{u}))$ is the orthogonal complement space of $\Psi(f(\widehat{u}))$;

$g_{n}(f(\widehat{u}))$ denotes the element in $\Psi_{n}^{\perp}(f(\widehat{u}))$, pick $g_{n}(f(\widehat{u}))=L^{*} f(\widehat{u})-P_{n} L^{*} f(\widehat{u})$;

$g(f(\widehat{u}))$ denotes the element in $\Psi^{\perp}(f(\widehat{u}))$, pick $g(f(\widehat{u}))=L^{*} f(\widehat{u})-P L^{*} f(\widehat{u})$.

It is easy to obtain the following lemmas.

Lemma 3.1. Suppose that $\left\{x_{i}\right\}_{i=1}^{\infty}$ is dense on $[0,1]$, then

$\left(1^{0}\right)\left\{\bar{\varphi}_{i}(x)\right\}_{i=0}^{\infty}$ is a complete system of $W_{1}$;

$\left(2^{0}\right)\left\{\bar{\varphi}_{i}(x)\right\}_{i=1}^{\infty}$ is not a complete system of $W_{1}$;

$\left(3^{0}\right)\left\{\psi_{i}(x)\right\}_{i=0}^{\infty}$ is a complete system of $W_{2}$;

$\left(4^{0}\right)\left\{\psi_{i}(x)\right\}_{i=1}^{\infty}$ is not a complete system of $W_{2}$.

Theorem 3.2. $u$ is the solution of (3.4) if and only if

$$
\begin{gathered}
\left\langle u, \psi_{0}(f(\widehat{u}))\right\rangle_{W_{2}}=\|f(\widehat{u})\|_{W_{1}}^{2} \\
\left\langle u, \psi_{i}(f(\widehat{u}))\right\rangle_{W_{2}}=0, \quad i=1,2, \ldots
\end{gathered}
$$


Proof. (Sufficiency)

From $\left\langle u, \psi_{0}(f(\widehat{u}))\right\rangle_{W_{2}}=\|f(\widehat{u})\|_{W_{1}}^{2}$, it follows that

$$
\left\langle L u, \bar{\varphi}_{0}\right\rangle_{W_{1}}=\left\langle u, L^{*} \bar{\varphi}_{0}\right\rangle_{W_{2}}=\left\langle u, \psi_{0}\right\rangle_{W_{2}}=\|f(\widehat{u})\|_{W_{1}}^{2}=\langle f(\widehat{u}), f(\widehat{u})\rangle_{W_{1}}=\left\langle f(\widehat{u}), \bar{\varphi}_{0}\right\rangle_{W_{1}} .
$$

From $\left\langle u, \psi_{i}(f(\widehat{u}))\right\rangle_{W_{2}}=0, i=1,2, \ldots$ and $\left\langle\bar{\varphi}_{0}, \bar{\varphi}_{i}\right\rangle_{W_{1}}=0, i \neq 0$, we have

$$
\left\langle L u, \bar{\varphi}_{i}\right\rangle_{W_{1}}=\left\langle u, L^{*} \bar{\varphi}_{i}\right\rangle_{W_{2}}=\left\langle u, \psi_{i}\right\rangle_{W_{2}}=0=\left\langle\bar{\varphi}_{0}, \bar{\varphi}_{i}\right\rangle_{W_{1}}=\left\langle f(\widehat{u}), \bar{\varphi}_{i}\right\rangle_{W_{1}}
$$

By the completeness of $\left\{\bar{\varphi}_{i}(x)\right\}_{i=0}^{\infty}$ and (3.7), (3.8), one obtains $L u=f(\widehat{u})$.

(Necessity)

If $u$ is the solution of (3.4), then

$$
\left\langle L u-f(\widehat{u}), \bar{\varphi}_{i}\right\rangle_{W_{1}}=0, \quad i=0,1,2, \ldots
$$

When $i=0$, we have

$$
\left\langle u, \psi_{0}\right\rangle_{W_{2}}=\left\langle L u, \bar{\varphi}_{0}\right\rangle_{W_{1}}=\left\langle f(\widehat{u}), \bar{\varphi}_{0}\right\rangle_{W_{1}}=\|f(\widehat{u})\|_{W_{1}}^{2} .
$$

When $i=1,2, \ldots$, we have

$$
\left\langle u, \psi_{i}\right\rangle_{W_{2}}=\left\langle L u, \bar{\varphi}_{i}\right\rangle_{W_{1}}=\left\langle\bar{\varphi}_{0}, \bar{\varphi}_{i}\right\rangle_{W_{1}}=0 .
$$

(3.10) and (3.11) yield (3.6).

In the same way, we can prove the following corollary.

Corollary 3.3. $u$ is the solution of (3.4) if and only if

$$
u=\frac{g(f(\widehat{u}))\|f(\widehat{u})\|_{W_{1}}^{2}}{\left\langle\psi_{0}(f(\widehat{u})), g(f(\widehat{u}))\right\rangle_{W_{2}}} .
$$




\section{Iterative Formulas}

\subsection{Iterative Formula I}

Pick arbitrarily initial function $\widehat{u}=u_{0}$ in (3.4) and denote the solution of (3.4) by $u_{n+1}$. Then, applying Corollary 3.3, one sees that

$$
\begin{gathered}
u_{1}=\frac{g\left(f\left(u_{0}\right)\right)\left\|f\left(u_{0}\right)\right\|_{W_{1}}^{2}}{\left\langle\psi_{0}\left(f\left(u_{0}\right)\right), g\left(f\left(u_{0}\right)\right)\right\rangle_{W_{2}}}, \\
u_{2}=\frac{g\left(f\left(u_{1}\right)\right)\left\|f\left(u_{1}\right)\right\|_{W_{1}}^{2}}{\left\langle\psi_{0}\left(f\left(u_{1}\right)\right), g\left(f\left(u_{1}\right)\right)\right\rangle_{W_{2}}}, \\
\vdots \\
u_{n+1}=\frac{g\left(f\left(u_{n}\right)\right)\left\|f\left(u_{n}\right)\right\|_{W_{1}}^{2}}{\left\langle\psi_{0}\left(f\left(u_{n}\right)\right), g\left(f\left(u_{n}\right)\right)\right\rangle_{W_{2}}},
\end{gathered}
$$

where

$$
g\left(f\left(u_{n}\right)\right)=L^{*} f\left(u_{n}\right)-P L^{*} f\left(u_{n}\right) \in \Psi^{\perp}\left(f\left(u_{n}\right)\right) .
$$

This is the iterative formula I. However, $P L^{*} f(\widehat{u})$ cannot be computed through finite steps. Thus, we will revise the formula.

\subsection{Iterative Formula II}

Replacing $g$ with $g_{n}$ in iterative formula I, we derive iterative formula II, that is

$$
u_{n+1}=\frac{g_{n}\left(f\left(u_{n}\right)\right)\left\|f\left(u_{n}\right)\right\|_{W_{1}}^{2}}{\left\langle\psi_{0}\left(f\left(u_{n}\right)\right), g_{n}\left(f\left(u_{n}\right)\right)\right\rangle_{W_{2}}},
$$

where

$$
g_{n}\left(f\left(u_{n}\right)\right)=L^{*} f\left(u_{n}\right)-P_{n} L^{*} f\left(u_{\mathrm{n}}\right) \in \Psi_{n}^{\perp}\left(f\left(u_{n}\right)\right), \quad n=0,1,2, \ldots
$$

Lemma 4.1. Let $\left.\bar{f}\left(u_{n}\right)\right) \triangleq f\left(u_{n}\right) /\left\|f\left(u_{n}\right)\right\|_{W_{1}}$ and

$$
\begin{aligned}
\beta & =\inf _{n}\left\|\psi_{0}\left(\bar{f}\left(u_{n}\right)\right)-P \psi_{0}\left(\bar{f}\left(u_{n}\right)\right)\right\|_{W_{2}} \\
& =\left\|\psi_{0}\left(f^{*}\right)-P \psi_{0}\left(f^{*}\right)\right\|_{W_{2}}
\end{aligned}
$$

then $\left\langle\psi_{0}\left(\bar{f}\left(u_{n}\right)\right), g_{n}\left(\bar{f}\left(u_{n}\right)\right)\right\rangle_{W_{2}} \geq \beta^{2}>0$. 
Proof. Note that

$$
\begin{aligned}
& \left\|\psi_{0}\left(\bar{f}\left(u_{n}\right)\right)-P_{n} \psi_{0}\left(\bar{f}\left(u_{n}\right)\right)\right\|_{W_{2}}^{2} \\
& \quad=\left\|\psi_{0}\left(\bar{f}\left(u_{n}\right)\right)-P \psi_{0}\left(\bar{f}\left(u_{n}\right)\right)+P \psi_{0}\left(\bar{f}\left(u_{n}\right)\right)-P_{n} \psi_{0}\left(\bar{f}\left(u_{n}\right)\right)\right\|_{W_{2}{ }^{\prime}}^{2}
\end{aligned}
$$

since

$$
\psi_{0}\left(\bar{f}\left(u_{n}\right)\right)-P \psi_{0}\left(\bar{f}\left(u_{n}\right)\right) \in \Psi^{\perp}\left(\bar{f}\left(u_{n}\right)\right), \quad P \psi_{0}\left(\bar{f}\left(u_{n}\right)\right)-P_{n} \psi_{0}\left(\bar{f}\left(u_{n}\right)\right) \in \Psi\left(\bar{f}\left(v_{n}\right)\right),
$$

this gives us

$$
\psi_{0}\left(\bar{f}\left(u_{n}\right)\right)-P \psi_{0}\left(\bar{f}\left(u_{n}\right)\right) \perp P \psi_{0}\left(\bar{f}\left(u_{n}\right)\right)-P_{n} \psi_{0}\left(\bar{f}\left(u_{n}\right)\right) .
$$

Thus, (4.6) leads to

$$
\begin{aligned}
\left\|\psi_{0}\left(\bar{f}\left(u_{n}\right)\right)-P_{n} \psi_{0}\left(\bar{f}\left(u_{n}\right)\right)\right\|_{W_{2}}^{2} & \left\|\psi_{0}\left(\bar{f}\left(u_{n}\right)\right)-P \psi_{0}\left(\bar{f}\left(u_{n}\right)\right)\right\|_{W_{2}}^{2} \\
& +\left\|P \psi_{0}\left(\bar{f}\left(u_{n}\right)\right)-P_{n} \psi_{0}\left(\bar{f}\left(u_{n}\right)\right)\right\|_{W_{2}}^{2} \\
\geq & \left\|\psi_{0}\left(\bar{f}\left(u_{n}\right)\right)-P \psi_{0}\left(\bar{f}\left(u_{n}\right)\right)\right\|_{W_{2}}^{2} \\
\geq & \left\|\psi_{0}\left(f^{*}\right)-P \psi_{0}\left(f^{*}\right)\right\|_{W_{2}}^{2} \\
= & \beta^{2} .
\end{aligned}
$$

However,

$$
\begin{aligned}
\beta^{2}= & \left\|\psi_{0}\left(f^{*}\right)-P \psi_{0}\left(f^{*}\right)\right\|_{W_{2}}^{2} \\
\leq & \left\|\psi_{0}\left(\bar{f}\left(u_{n}\right)\right)-P \psi_{0}\left(\bar{f}\left(u_{n}\right)\right)\right\|_{W_{2}}^{2} \leq\left\|\psi_{0}\left(\bar{f}\left(u_{n}\right)\right)-P_{n} \psi_{0}\left(\bar{f}\left(u_{n}\right)\right)\right\|_{W_{2}}^{2} \\
= & \left\langle\psi_{0}\left(\bar{f}\left(u_{n}\right)\right)-P_{n} \psi_{0}\left(\bar{f}\left(v_{n}\right)\right), \psi_{0}\left(\bar{f}\left(u_{n}\right)\right)-P_{n} \psi_{0}\left(\bar{f}\left(u_{n}\right)\right)\right\rangle_{W_{2}} \\
= & \left\langle\psi_{0}\left(\bar{f}\left(u_{n}\right)\right), \psi_{0}\left(\bar{f}\left(u_{n}\right)\right)-P_{n} \psi_{0}\left(\bar{f}\left(u_{n}\right)\right)\right\rangle_{W_{2}} \\
& -\left\langle P_{n} \psi_{0}\left(\bar{f}\left(u_{n}\right)\right), \psi_{0}\left(\bar{f}\left(u_{n}\right)\right)-P_{n} \psi_{0}\left(\bar{f}\left(u_{n}\right)\right)\right\rangle_{W_{2}} .
\end{aligned}
$$

Because $\left\langle P_{n} \psi_{0}\left(\bar{f}\left(u_{n}\right)\right), \psi_{0}\left(\bar{f}\left(u_{n}\right)\right)-P_{n} \psi_{0}\left(\bar{f}\left(u_{n}\right)\right)\right\rangle_{W_{2}}=0$, hence

$$
\beta^{2} \leq\left\langle\psi_{0}\left(\bar{f}\left(u_{n}\right)\right), g_{n}\left(\bar{f}\left(u_{n}\right)\right)\right\rangle_{W_{2}}
$$


Now, we prove $\beta>0$. Suppose that $\beta=0$, then (4.5) gives us

$$
\begin{aligned}
0 & =\left\|\psi_{0}\left(f^{*}\right)-P \psi_{0}\left(f^{*}\right)\right\|_{W_{2}}^{2} \\
& =\left\langle\psi_{0}\left(f^{*}\right)-P \psi_{0}\left(f^{*}\right), \psi_{0}\left(f^{*}\right)-P \psi_{0}\left(f^{*}\right)\right\rangle_{W_{2}} \\
& =\left\langle\psi_{0}\left(f^{*}\right)-P \psi_{0}\left(f^{*}\right), g\left(f^{*}\right)\right\rangle_{W_{2}} \\
& =\left\langle\psi_{0}\left(f^{*}\right), g\left(f^{*}\right)\right\rangle_{W_{2}}-\left\langle P \psi_{0}\left(f^{*}\right), g\left(f^{*}\right)\right\rangle_{W_{2}} .
\end{aligned}
$$

Since $g\left(f^{*}\right)=L^{*} f^{*}-P L^{*} f^{*} \in \Psi^{\perp}\left(f^{*}\right)$ and $P \psi_{0}\left(f^{*}\right)=P L^{*} f^{*} \in \Psi\left(f^{*}\right)$, hence $\left\langle P \psi_{0}\left(f^{*}\right), g\left(f^{*}\right)\right\rangle_{W_{2}}=0$, furthermore, $\left\langle\psi_{0}\left(f^{*}\right), g\left(f^{*}\right)\right\rangle_{W_{2}}=0$, namely, $\psi_{0}\left(f^{*}\right) \perp g\left(f^{*}\right)$. Note here that $g\left(f^{*}\right) \in \Psi^{\perp}\left(f^{*}\right)$, one has

$$
\psi_{0}\left(f^{*}\right) \in \Psi\left(f^{*}\right)=\operatorname{Span}\left\{\psi_{1}\left(f^{*}\right), \psi_{2}\left(f^{*}\right), \ldots\right\}
$$

From $\left(3^{0}\right)$ of Lemma 3.1, we know that $\left\{\psi_{i}(x)\right\}_{i=0}^{\infty}$ is a complete system of $W_{2}[0,1]$. On the other hand, by $\psi_{0}\left(f^{*}\right) \in \Psi\left(f^{*}\right)$, we obtain $\left\{\psi_{i}(x)\right\}_{i=1}^{\infty}$ is a complete system of $W_{2}[0,1]$. This is a contradiction to $\left(4^{0}\right)$ of Lemma 3.1, thus, $\beta>0$.

From Lemma 4.1 we know that iterative formula is significative.

\section{Existence of Solution for (3.3)}

Put $\widehat{u}=u_{n}$ in (3.4), then by Corollary 3.3, $L u=f\left(u_{n}\right)$ can be written by

$$
u=\frac{g\left(f\left(u_{n}\right)\right)\left\|f\left(u_{n}\right)\right\|^{2}}{\left\langle\psi_{0}\left(f\left(u_{n}\right)\right), g\left(f\left(u_{n}\right)\right)\right\rangle_{W_{2}}}
$$

Replacing $u$ with $v_{n}$, then

$$
\begin{gathered}
L v_{n}=f\left(u_{n}\right), \\
v_{n}=\frac{g\left(f\left(u_{n}\right)\right)\left\|f\left(u_{n}\right)\right\|^{2}}{\left\langle\psi_{0}\left(f\left(u_{n}\right)\right), g\left(f\left(u_{n}\right)\right)\right\rangle_{W_{2}}} .
\end{gathered}
$$

Obviously, iterative formula II can be converted into the form as follows:

$$
\begin{gathered}
L v_{n}=f\left(u_{n}\right), \\
u_{n+1}=\alpha_{n} v_{n}, \\
v_{n}(1)=v_{n}^{\prime}(0)=v_{n}^{\prime}(1)=0, \quad n \in N^{+},
\end{gathered}
$$


where

$$
\begin{gathered}
\alpha_{n}=\frac{\left\langle\psi_{0}\left(f\left(u_{n}\right)\right), g\left(f\left(u_{n}\right)\right)\right\rangle_{W_{2}}}{\left\langle\psi_{0}\left(f\left(u_{n}\right)\right), g_{n}\left(f\left(u_{n}\right)\right)\right\rangle_{W_{2}}} \cdot \frac{g_{n}\left(f\left(u_{n}\right)\right)}{g\left(f\left(u_{n}\right)\right)}, \\
g_{n}\left(f\left(u_{n}\right)\right)=L^{*} f\left(u_{n}\right)-P_{n} L^{*} f\left(u_{n}\right) \in \Psi_{n}^{\perp} \\
g\left(f\left(u_{n}\right)\right)=L^{*} f\left(u_{n}\right)-P L^{*} f\left(u_{n}\right) \in \Psi^{\perp} .
\end{gathered}
$$

Note that by Lemma 4.1 we know

$$
\left\langle\psi_{0}\left(f\left(u_{n}\right)\right), g_{n}\left(f\left(u_{n}\right)\right)\right\rangle_{W_{2}}=\left\|f\left(u_{n}\right)\right\|_{W_{1}}^{2}\left\langle\psi_{0}\left(\bar{f}\left(u_{n}\right)\right), g_{n}\left(\bar{f}\left(u_{n}\right)\right)\right\rangle_{W_{2}} \geq\left\|f\left(u_{n}\right)\right\|_{W_{1}}^{2} \beta^{2}>0
$$

in (5.5). Now suppose $g\left(f\left(u_{n}(x)\right) \neq 0\right.$, if there exists an $x$, such that $g\left(f\left(u_{n}(x)\right)=0\right.$, then we will change definition of $\alpha_{n}(x)$ :

$$
\alpha_{n}(x)=\frac{\left\langle\psi_{0}\left(f\left(u_{n}\right)\right), g\left(f\left(u_{n}\right)\right)\right\rangle_{W_{2}}}{\left\langle\psi_{0}\left(f\left(u_{n}\right)\right), g_{n}\left(f\left(u_{n}\right)\right)\right\rangle_{W_{2}}} \cdot \frac{g_{n}\left(f\left(u_{n}\right)\right)+M}{g\left(f\left(u_{n}\right)\right)+M}
$$

where $M$ satisfies $g\left(f\left(u_{n}\right)\right)+M \geq \alpha>0$.

Lemma 5.1. A bounded set in $W_{1}$ is a compacted set in $C[0,1]$.

Lemma 5.2. For $u \in W_{2}, v \in W_{1}$, one has

$$
\begin{gathered}
\left|u^{(i)}(x)\right| \leq \mu_{i}\|u\|_{W_{2}}, \quad i=0,1,2,3, \\
|v(x)| \leq \mu\|v\|_{W_{1}} .
\end{gathered}
$$

Lemma 5.3. A bounded set in $W_{2}$ is a compacted set in $C^{3}[0,1]$.

Lemma 5.4. Let $\left.\bar{f}\left(u_{n}\right)\right) \triangleq f\left(u_{n}\right) /\left\|f\left(u_{n}\right)\right\|_{W_{1}}$, then $\left\|\alpha_{n}-1\right\|_{C^{3}} \rightarrow 0,(n \rightarrow \infty)$.

Proof. (1) Since

$$
\begin{aligned}
\left\|\psi_{0}\left(\bar{f}\left(u_{n}\right)\right)\right\|_{W_{2}} & =\left\|\psi_{0}\left(\frac{f\left(u_{n}\right)}{\left\|f\left(u_{n}\right)\right\|_{W_{1}}}\right)\right\|_{W_{2}} \\
& =\frac{1}{\left\|f\left(u_{n}\right)\right\|_{W_{1}}}\left\|\psi_{0}\left(f\left(u_{n}\right)\right)\right\|_{W_{2}} \\
& =\frac{1}{\left\|f\left(u_{n}\right)\right\|_{W_{1}}}\left\|L^{*} f\left(u_{n}\right)\right\|_{W_{2}} \\
& \leq \frac{1}{\left\|f\left(u_{n}\right)\right\|_{W_{1}}}\left\|f\left(u_{n}\right)\right\|_{W_{1}}\|L\|=\|L\|,
\end{aligned}
$$


Boundary Value Problems

11

$$
\begin{aligned}
\left\|g\left(\bar{f}\left(u_{n}\right)\right)-g_{n}\left(\bar{f}\left(u_{n}\right)\right)\right\|_{W_{2}} & =\frac{1}{\left\|f\left(u_{n}\right)\right\|_{W_{1}}}\left\|g\left(f\left(u_{n}\right)\right)-g_{n}\left(f\left(u_{n}\right)\right)\right\|_{W_{2}} \\
& =\frac{1}{\left\|f\left(u_{n}\right)\right\|_{W_{1}}}\left\|P L^{*}\left(f\left(u_{n}\right)\right)-P_{n} L^{*} f\left(\left(u_{n}\right)\right)\right\|_{W_{2}} \\
& \leq \frac{1}{\left\|f\left(u_{n}\right)\right\|_{W_{1}}}\left\|P-P_{n}\right\|\left\|L^{*} f\left(u_{n}\right)\right\| \\
& \leq \frac{1}{\left\|f\left(u_{n}\right)\right\|_{W_{1}}}\left\|f\left(u_{n}\right)\right\|_{W_{1}}\|L\|\left\|P-P_{n}\right\| \longrightarrow 0, \quad(n \longrightarrow \infty),
\end{aligned}
$$

hence, by Lemma 4.1, one gets

$$
\begin{aligned}
& \left|\frac{\left\langle\psi_{0}\left(f\left(u_{n}\right)\right), g\left(f\left(u_{n}\right)\right)\right\rangle_{W_{2}}}{\left\langle\psi_{0}\left(f\left(u_{n}\right)\right), g_{n}\left(f\left(u_{n}\right)\right)\right\rangle_{W_{2}}}-1\right| \\
& \quad=\left|\frac{\left\langle\psi_{0}\left(\bar{f}\left(u_{n}\right)\right), g\left(\bar{f}\left(u_{n}\right)\right)\right\rangle_{W_{2}}}{\left\langle\psi_{0}\left(\bar{f}\left(u_{n}\right)\right), g_{n}\left(\bar{f}\left(u_{n}\right)\right)\right\rangle_{W_{2}}}-1\right| \\
& \quad=\mid \frac{\left\langle\psi_{0}\left(\bar{f}\left(u_{n}\right)\right), g\left(\bar{f}\left(u_{n}\right)\right)-g_{n}\left(\bar{f}\left(u_{n}\right)\right)\right\rangle_{W_{2}}}{\left\langle\psi_{0}\left(\bar{f}\left(u_{n}\right)\right), g_{n}\left(\bar{f}\left(u_{n}\right)\right)\right\rangle_{W_{2}} \mid} \\
& \quad \leq \frac{1}{\beta^{2}}\left|\left\langle\psi_{0}\left(\bar{f}\left(u_{n}\right)\right), g\left(\bar{f}\left(u_{n}\right)\right)-g_{n}\left(\bar{f}\left(u_{n}\right)\right)\right\rangle_{W_{2}}\right|, \quad(\beta>0) \\
& \leq \frac{1}{\beta^{2}}\left\|\psi_{0}\left(\bar{f}\left(u_{n}\right)\right)\right\|_{W_{2}}\left\|g\left(\bar{f}\left(u_{n}\right)\right)-g_{n}\left(\bar{f}\left(u_{n}\right)\right)\right\|_{W_{2}} \rightarrow 0, \quad(n \rightarrow \infty),
\end{aligned}
$$

namely,

$$
\frac{\left\langle\psi_{0}\left(f\left(u_{n}\right)\right), g\left(f\left(u_{n}\right)\right)\right\rangle_{W_{2}}}{\left\langle\psi_{0}\left(f\left(u_{n}\right)\right), g_{n}\left(f\left(u_{n}\right)\right)\right\rangle_{W_{2}}} \rightarrow 1 \quad(n \rightarrow \infty) \text {. }
$$

(2) Note that

$$
\begin{aligned}
\left\|g\left(\bar{f}\left(u_{n_{k}}\right)\right)\right\|_{W_{2}} & =\left\|L^{*}\left(\bar{f}\left(u_{n_{k}}\right)\right)-P L^{*}\left(\bar{f}\left(u_{n_{k}}\right)\right)\right\|_{W_{2}} \\
& \leq\left\|L^{*}\left(\bar{f}\left(u_{n_{k}}\right)\right)\right\|_{W_{2}}+\left\|P L^{*}\left(\bar{f}\left(u_{n_{k}}\right)\right)\right\|_{W_{2}} \leq 2\|L\|, \\
\left\|g_{n_{k}}\left(\bar{f}\left(u_{n_{k}}\right)\right)\right\|_{W_{2}} & =\left\|L^{*}\left(\bar{f}\left(u_{n_{k}}\right)\right)-P_{n_{k}} L^{*}\left(\bar{f}\left(u_{n_{k}}\right)\right)\right\|_{W_{2}} \\
& \leq\left\|L^{*}\left(\bar{f}\left(u_{n_{k}}\right)\right)\right\|_{W_{2}}+\left\|P_{n_{k}} L^{*}\left(\bar{f}\left(u_{n_{k}}\right)\right)\right\|_{W_{2}} \leq 2\|L\|,
\end{aligned}
$$


thus, by Lemma 5.3, there exists $\left\{n_{k_{j}}\right\}_{j=1}^{\infty}$ of $\left\{n_{\mathrm{k}}\right\}_{k=1}^{\infty}$, such that

$$
g_{n_{k_{j}}}\left(\bar{f}\left(u_{n_{k_{j}}}\right)\right) \stackrel{C^{3}}{\longrightarrow} g^{*}(x) \neq 0 .
$$

By (5.11), it follows that

$$
\begin{aligned}
\left\|g_{n_{k_{j}}}\left(\bar{f}\left(u_{n_{k_{j}}}\right)\right)-g\left(\bar{f}\left(u_{n_{k_{j}}}\right)\right)\right\|_{C^{3}} & \leq M\left\|g_{n_{k_{j}}}\left(\bar{f}\left(u_{n_{k_{j}}}\right)\right)-g\left(\bar{f}\left(u_{n_{k_{j}}}\right)\right)\right\|_{W_{2}} \\
& =M\left\|g_{n}\left(\bar{f}\left(u_{n}\right)\right)-g\left(\bar{f}\left(u_{n}\right)\right)\right\|_{W_{2}} \longrightarrow 0, \quad(n \longrightarrow \infty) .
\end{aligned}
$$

Therefore,

$$
\begin{aligned}
\varlimsup_{n \rightarrow \infty}\left\|\frac{g_{n}\left(f\left(u_{n}\right)\right)}{g\left(f\left(u_{n}\right)\right)}-1\right\|_{C^{3}} & =\varlimsup_{n \rightarrow \infty}\left\|\frac{g_{n}\left(\bar{f}\left(u_{n}\right)\right)}{g\left(\bar{f}\left(u_{n}\right)\right)}-1\right\|_{C^{3}} \\
& =\varlimsup_{n \rightarrow \infty}\left\|\frac{g_{n}\left(\bar{f}\left(u_{n}\right)\right)-g\left(\bar{f}\left(u_{n}\right)\right)}{g\left(\bar{f}\left(u_{n}\right)\right)}\right\|_{C^{3}} \\
& =\lim _{k \rightarrow \infty}\left\|\frac{g_{n_{k}}\left(\bar{f}\left(u_{n_{k}}\right)\right)-g\left(\bar{f}\left(u_{n_{k}}\right)\right)}{g\left(\bar{f}\left(u_{n_{k}}\right)\right)}\right\|_{C^{3}} \\
& =\| \frac{\lim _{j \rightarrow \infty}\left\|g_{n_{k_{j}}}\left(\bar{f}\left(u_{n_{k_{j}}}\right)\right)-g\left(\bar{f}\left(u_{n_{k_{j}}}\right)\right)\right\| \|_{C^{3}}}{\lim _{j \rightarrow \infty} g\left(\bar{f}\left(u_{n_{k_{j}}}\right)\right)} \\
& =\frac{1}{\left\|g^{*}(x)\right\|_{C^{3}}} \lim _{j \rightarrow \infty}\left\|g_{n_{k_{j}}}\left(\bar{f}\left(u_{n_{k_{j}}}\right)\right)-g\left(\bar{f}\left(u_{n_{k_{j}}}\right)\right)\right\|_{C^{3}} . \\
& =0 .
\end{aligned}
$$

In the similar manner, one gets

$$
\frac{\lim }{n}\left\|\frac{g_{n}\left(f\left(u_{n}\right)\right)}{g\left(f\left(u_{n}\right)\right)}-1\right\|_{C^{3}}=0 .
$$

Combining the above argument, we obtain

$$
\lim _{n}\left\|\frac{g_{n}\left(f\left(u_{n}\right)\right)}{g\left(f\left(u_{n}\right)\right)}-1\right\|_{C^{3}}=0 .
$$

The lemma is complete from (5.13) and (5.20). 
Lemma 5.5. $\left\|u_{n}\right\|_{W_{2}} \leq M\left\|v_{n}\right\|_{W_{2}}$.

Lemma 5.6. If $f(x, y, z, w)$ satisfies condition $H$, then $\left\|v_{n}\right\|_{W_{2}},\left\|u_{n}\right\|_{W_{2}}$ are bounded. (Technique of proof is similar to the Lemma 7.1 in appendix).

Lemma 5.7. If $\theta_{n} \in(0,1)$ and $v_{n}^{\prime \prime}(\theta n)=0$, then $\theta_{n}$ does not infinitely go closer to 1 , as there exists $\theta \in(0,1)$, such that $\theta_{n}<\theta<1$.

Proof. Since $v_{n}^{\prime}(0)=0$ and $v_{n}^{\prime}(1)=0$, there exists $\theta_{n} \in(0,1)$, such that $v_{n}^{\prime \prime}(\theta n)=0$.

Suppose $\theta_{n} \rightarrow 1$, as $n \rightarrow \infty$. By Lemmas 5.3 and 5.6, there exists $v(x)$ such that $\left\|v_{n}-v\right\|_{C^{3}} \rightarrow 0$; thus $\left|v_{n}(x)-v(x)\right| \rightarrow 0,\left|v_{n}^{\prime}(x)-v^{\prime}(x)\right| \rightarrow 0,\left|v_{n}^{\prime \prime}(x)-v^{\prime \prime}(x)\right| \rightarrow 0$. Since $v_{n}^{\prime}(0)=0, v_{n}^{\prime}(1)=0, v_{n}^{\prime \prime}(\theta n)=0$, it follows that $v^{\prime}(0)=0, v^{\prime}(1)=0, v^{\prime \prime}(1)=0$. Hence there exists an $\eta \in(0,1)$, such that $v^{\prime \prime}(\eta)=0$, thus there exists an $\xi \in(\eta, 1)$, such that $v^{(3)}(\xi)=0$. By (5.4), $L v_{n}(x)=f\left(x, u_{n}(x), u_{n}^{\prime}(x), u_{n}^{\prime \prime}(x)\right)$, taking limit for $n$ on both sides, we have

$$
L v(x)=f\left(x, u(x), u^{\prime}(x), u^{\prime \prime}(x)\right),
$$

thus $f\left(\xi, u(\xi), u^{\prime}(\xi), u^{\prime \prime}(\xi)\right)=v^{(3)}(\xi)=0$; this is a contradiction to $(\mathrm{H} 3)$.

Lemma 5.8. If $f(x, y, z, w)$ satisfies condition $H$, then $\left\|u_{n+1}-u_{n}\right\|_{C^{2}} \rightarrow 0$ as $n \rightarrow \infty$. (see Lemma 7.3 in the appendix)

Theorem 5.9 (Existence). If $f(x, y, z, w)$ satisfies condition $H$, then the solution of (1.2) exists in $W_{2}[0,1]$.

Proof. From Lemma 5.6, $\left\|u_{n}\right\|_{W_{3}} \leq M$, so by Lemma 5.3, $\left\{u_{n}(x)\right\}$ is compact in $C^{3}[0,1]$, then there exists convergent subsequence $\left\{u_{n_{k}}(x)\right\}$ of $\left\{u_{n}(x)\right\}$, such that $\left\|u_{n_{k}}-u\right\|_{C^{3}} \rightarrow 0$, in the similar manner, there exists $v(x)$, such that $\left\|v_{n_{k}}-v\right\|_{C^{3}} \rightarrow 0$, thus $\left|u_{n_{k}}(x)-u(x)\right| \rightarrow 0$, $\left|v_{n_{k}}(x)-v(x)\right| \rightarrow 0$, as $k \rightarrow \infty$.

By Lemma 5.4, $\left|\alpha_{n_{k}}-1\right| \rightarrow 0$, as $k \rightarrow \infty$ and by Lemma 5.8, $\left|u_{n_{k}+1}(x)-u_{n_{k}}(x)\right| \rightarrow 0$, then $\left|u_{n_{k}+1}(x)-u(x)\right| \leq\left|u_{n_{k}+1}(x)-u_{n_{k}}(x)\right|+\left|u_{n_{k}}(x)-u(x)\right| \rightarrow 0$; thus

$$
v(x)=\lim _{k \rightarrow \infty} v_{n_{k}}(x)=\lim _{k \rightarrow \infty} \frac{1}{\alpha_{n_{k}}} u_{n_{k}+1}=\lim _{k \rightarrow \infty} \frac{1}{\alpha_{n_{k}}} \cdot \lim _{k \rightarrow \infty} u_{n_{k}+1}=1 \cdot \lim _{k \rightarrow \infty} u_{n_{k}}=u(x) .
$$

From (5.4) and (5.22), $L u(x)=f\left(x, u(x), u^{\prime}(x), u^{\prime \prime}(x)\right)$, the solution of (1.2) exists. Since $\mathrm{f}\left(x, u(x), u^{\prime}(x), u^{\prime \prime}(x)\right) \in W_{1}[0,1]$, thus $u(x) \in W_{2}[0,1]$.

Theorem 5.10. If $f(x, y, z, w)$ satisfies condition $H$ and the solution $u(x)$ of (1.2) is unique, then the sequence $\left\{u_{n}(x)\right\}_{n=1}^{\infty}$ is convergent to $u(x)$, and $\left\|u_{n}^{(k)}(x)-u^{(k)}(x)\right\|_{C} \rightarrow 0, k=0,1,2,3$.

Proof. Suppose that $\left\{u_{n}(x)\right\}_{n=1}^{\infty}$ is not convergent to $u(x)$, since $\left\|u_{n}\right\|_{W_{2}}$ is bounded, we can choose two subsequences $\left\{u_{n_{k}}(x)\right\}_{k=1}^{\infty},\left\{u_{n_{j}}(x)\right\}_{j=1}^{\infty}$ of $\left\{u_{n}(x)\right\}_{n=1}^{\infty}$ such that $u_{n_{k}}(x) \rightarrow u_{1}(x)$, $u_{n_{j}}(x) \rightarrow u_{2}(x)$ and $u_{1}(x) \neq u_{2}(x)$, From Theorem 5.9, $u_{1}(x)$ and $u_{2}(x)$ are the solutions of (1.2), this contradicts the uniqueness of solution of (1.2). consequently, $\left\|u_{n}-u\right\|_{C} \rightarrow 0$.

In the same way, we can verify $\left\|u_{n}^{(k)}-u^{(k)}\right\|_{C} \rightarrow 0, n \rightarrow \infty, k=1,2,3$. 
Table 1: Relative errors $E(x)$ at $n=10$.

\begin{tabular}{lccccc}
\hline Node & $E(x)$ & Node & $E(x)$ & Node & $E(x)$ \\
\hline $1 / 10$ & $4.71412 \mathrm{E}-03$ & $1 / 5$ & $1.23304 \mathrm{E}-03$ & $3 / 10$ & $1.74306 \mathrm{E}-03$ \\
$2 / 5$ & $4.25259 \mathrm{E}-03$ & $1 / 2$ & $6.10987 \mathrm{E}-03$ & $3 / 5$ & $7.10274 \mathrm{E}-03$ \\
$7 / 10$ & $6.88828 \mathrm{E}-03$ & $4 / 5$ & $4.62244 \mathrm{E}-03$ & $9 / 10$ & $3.53199 \mathrm{E}-03$ \\
\hline
\end{tabular}

\section{Numerical Example}

Now we present a numerical example for solving (1.2) in the reproducing kernel space $W_{2}[0,1]$. All computations are performed by the Mathematica 5.0.

Example 6.1. Let us consider the following equation:

$$
\begin{gathered}
u^{3}(x)=x u^{\prime \prime}(x)+u^{\prime}(x)+x u(x)+u^{2}(x) \\
u(1)=u^{\prime}(0)=u^{\prime}(1)=0
\end{gathered}
$$

where $x \in[0,1]$. The true solution is $u(x)=x(x-1)$. Using our method, 10 points are chosen on $[0,1]$ and we calculate the relative errors $E(x) \stackrel{\text { def }}{=}\left\|\left(u(x)-u_{n}(x)\right) / u(x)\right\|_{C}$. The numerical results are presented in Table 1 which shows the method given in the paper is efficient.

\section{Appendix}

In order to obtain proofs of Lemma 5.6 and Lemma 5.8, firstly, we give an expression of solution of (1.1). For any initial value function $u_{0}(x) \in W_{3}[0,1]$,

$$
\begin{aligned}
v_{n}(x)= & \int_{0}^{x} d t \int_{0}^{t} d \eta \int_{0}^{\eta} f\left(\xi, u_{n-1}(\xi), u_{n-1}^{\prime}(\xi), u_{n-1}^{\prime \prime}(\xi)\right) d \xi \\
& -\frac{1}{2} x^{2} \int_{0}^{1} d \eta \int_{0}^{\eta} f\left(\xi, u_{n-1}(\xi), u_{n-1}^{\prime}(\xi), u_{n-1}^{\prime \prime}(\xi)\right) d \xi \\
u_{n}(x)= & \widetilde{P_{n}} v_{n}, \quad n=1,2, \ldots,
\end{aligned}
$$

where $\widetilde{P_{n}}: W_{3} \rightarrow \Psi_{n}(f(\widehat{u}))$ is a orthogonal projector.

Lemma 7.1. If $f(x, y, z, w)$ satisfies condition $H$, then $\left\|v_{n}\right\|_{W_{3}},\left\|u_{n}\right\|_{W_{3}}$ are bounded.

Proof. By (H2), $\left|f\left(x, u_{n-1}(x), u_{n-1}^{\prime}(x), u_{n-1}^{\prime \prime}(x)\right)\right| \leq M$, from (7.1),

$$
\begin{aligned}
& \left|v_{n}^{\prime}(x)\right| \\
& \quad=\left|\int_{0}^{x} d \eta \int_{0}^{\eta} f\left(\xi, u_{n-1}(\xi), u_{n-1}^{\prime}(\xi), u_{n-1}^{\prime \prime}(\xi)\right) d \xi-x \int_{0}^{1} d \eta \int_{0}^{\eta} f\left(\xi, u_{n-1}(\xi), u_{n-1}^{\prime}(\xi), u_{n-1}^{\prime \prime}(\xi)\right) d \xi\right|
\end{aligned}
$$




$$
\begin{aligned}
\leq & \int_{0}^{x} d \eta \int_{0}^{\eta}\left|f\left(\xi, u_{n-1}(\xi), u_{n-1}^{\prime}(\xi), u_{n-1}^{\prime \prime}(\xi)\right)\right| d \xi \\
& +|x| \int_{0}^{1} d \eta \int_{0}^{\eta}\left|f\left(\xi, u_{n-1}(\xi), u_{n-1}^{\prime}(\xi), u_{n-1}^{\prime \prime}(\xi)\right)\right| d \xi \\
\leq & M \frac{1}{2}\left|x^{2}\right|+|x| M \frac{1}{2} \leq M .
\end{aligned}
$$

Using this method, $\left|v_{n}^{\prime \prime}(x)\right| \leq(3 / 2) M,\left|v_{n}^{(3)}(x)\right| \leq M$. By Lemma 5.4, $\left|\alpha_{n}^{(i)}\right|, i=0,1,2,3$ are bounded, then $\left|u_{n}(x)\right|=\left|\alpha_{n-1} v_{n-1}(x)\right|,\left|u_{n}^{\prime}(x)\right|=\left|\alpha_{n-1}^{\prime} v_{n-1}+\alpha_{n-1} v_{n-1}^{\prime}\right|,\left|u_{n}^{\prime \prime}(x)\right|=\mid \alpha_{n-1}^{\prime \prime} v_{n-1}+$ $2 \alpha_{n-1}^{\prime} v_{n-1}^{\prime}+\alpha_{n-1} v_{n-1}^{\prime \prime} \mid$ and $\left|u_{n}^{(3)}(x)\right|=\left|\alpha_{n-1}^{(3)} v_{n-1}+3 \alpha_{n-1}^{\prime} v_{n-1}^{\prime \prime}+3 \alpha_{n-1}^{\prime \prime} v_{n-1}^{\prime}+\alpha_{n-1} v_{n-1}^{(3)}\right|$ are bounded, thus by (H2), $\left|v_{n}^{(4)}(x)\right|=\left|f_{x}+f_{y} u_{n-1}^{\prime}(x)+f_{z} u_{n-1}^{\prime \prime}(x)+f_{w} u_{n-1}^{(3)}(x)\right|$ is bounded. Consequently, we know that $\left\|v_{n}\right\|_{W_{3}}$ is bounded by the definition of $\|\cdot\|_{W_{3}}$, and by Lemma 5.5, $\left\|u_{n}\right\|_{W_{3}}$ is bounded.

Lemma 7.2. If $f(x, y, z, w)$ satisfies condition $H$, then $\left|v_{n+1}^{\prime \prime}(0)-v_{n}^{\prime \prime}(0)\right| \rightarrow 0,\left|v_{n+1}(0)-v_{n}(0)\right| \rightarrow 0$, as $n \rightarrow \infty$.

Proof. By Lemma 5.4, $\left\|\alpha_{n}\right\|_{C^{3}} \leq M_{1}$, and from Lemmas 5.2 and 5.6, $\left\|v_{n}\right\|_{C^{3}} \leq M_{2}$, then

$$
\begin{aligned}
\left|u_{n+1}(x)-u_{n}(x)\right| & =\left|\alpha_{n} v_{n}(x)-\alpha_{n-1} v_{n-1}(x)\right| \\
& =\left|\alpha_{n} v_{n}(x)-\alpha_{n} v_{n-1}+\alpha_{n} v_{n-1}-\alpha_{n-1} v_{n-1}(x)\right| \\
& \leq|| \alpha_{n}\left\|_{C_{3}}\left|v_{n}(x)-v_{n-1}(x)\right|+|| v_{n-1}\right\|_{C_{3}}\left|\alpha_{n}-\alpha_{n-1}\right| \\
& \leq M_{1}\left(\left|v_{n}(x)-v_{n-1}(x)\right|+M_{2}\left|\alpha_{n}-\alpha_{n-1}\right|\right) \\
& \leq M_{1}\left(\left|v_{n}(x)-v_{n-1}(x)\right|+\left|v_{n}^{\prime}(x)-v_{n-1}^{\prime}(x)\right|+\left|v_{n}^{\prime \prime}(x)-v_{n-1}^{\prime \prime}(x)\right|\right)+\rho_{n}
\end{aligned}
$$

where $\rho_{n}=M_{2}\left\|\alpha_{n}-\alpha_{n-1}\right\|_{C^{3}}$, by Lemma 5.4, $\left\|\alpha_{n}-\alpha_{n-1}\right\|_{C^{3}} \rightarrow 0$, then $\rho_{n} \rightarrow 0$.

In the similar manner,

$$
\begin{aligned}
& \left|u_{n+1}^{\prime}(x)-u_{n}^{\prime}(x)\right| \leq M_{1}\left(\left|v_{n}(x)-v_{n-1}(x)\right|+\left|v_{n}^{\prime}(x)-v_{n-1}^{\prime}(x)\right|+\left|v_{n}^{\prime \prime}(x)-v_{n-1}^{\prime \prime}(x)\right|\right)+\rho_{n} \\
& \left|u_{n+1}^{\prime \prime}(x)-u_{n}^{\prime \prime}(x)\right| \leq 2 M_{1}\left(\left|v_{n}(x)-v_{n-1}(x)\right|+\left|v_{n}^{\prime}(x)-v_{n-1}^{\prime}(x)\right|+\left|v_{n}^{\prime \prime}(x)-v_{n-1}^{\prime \prime}(x)\right|\right)+2 \rho_{n}
\end{aligned}
$$

$$
\begin{aligned}
& \left|u_{n+1}(x)-u_{n}(x)\right|+\left|u_{n+1}^{\prime}(x)-u_{n}^{\prime}(x)\right|+\left|u_{n+1}^{\prime \prime}(x)-u_{n}^{\prime \prime}(x)\right| \\
& \quad \leq 4 M_{1}\left(\left|v_{n}(x)-v_{n-1}(x)\right|+\left|v_{n}^{\prime}(x)-v_{n-1}^{\prime}(x)\right|+\left|v_{n}^{\prime \prime}(x)-v_{n-1}^{\prime \prime}(x)\right|\right)+4 \rho_{n} .
\end{aligned}
$$


Since $L v_{n}(x)=v_{n}^{(3)}(x)=f\left(x, u_{n-1}(x), u_{n-1}^{\prime}(x), u_{n-1}^{\prime \prime}(x)\right)$, then

$$
\begin{aligned}
& v_{n}^{\prime \prime}(x)=\int_{\theta_{n}}^{x} f\left(\xi, u_{n-1}(\xi), u_{n-1}^{\prime}(\xi), u_{n-1}^{\prime \prime}(\xi)\right) d \xi \\
& v_{n}^{\prime}(x)=\int_{0}^{x} d \eta \int_{\theta_{n}}^{\eta} f\left(\xi, u_{n-1}(\xi), u_{n-1}^{\prime}(\xi), u_{n-1}^{\prime \prime}(\xi)\right) d \xi \\
& v_{n}(x)=\int_{0}^{x} d t \int_{0}^{t} d \eta \int_{\theta_{n}}^{\eta} f\left(\xi, u_{n-1}(\xi), u_{n-1}^{\prime}(\xi), u_{n-1}^{\prime \prime}(\xi)\right) d \xi
\end{aligned}
$$

where $\theta_{n} \in(0,1)$ and $v_{n}^{\prime \prime}(\theta n)=0$. When $x \in(\theta, 1], \theta$ is in Lemma 5.7, from $\mathrm{H}(2)$, let $M_{3}=$ $\max \left\{f_{y}, f_{z}, f_{w}\right\}$,

$$
\begin{aligned}
\left|v_{n+1}(x)-v_{n}(x)\right| \leq & \mid \int_{0}^{x} d t \int_{0}^{t} d \eta \int_{\theta_{n+1}}^{\eta} f\left(\xi, u_{n}(\xi), u_{n}^{\prime}(\xi), u_{n}^{\prime \prime}(\xi)\right) d \xi \\
\leq & -\int_{0}^{x} d t \int_{0}^{t} d \eta \int_{\theta_{n}}^{\eta} d t \int_{0}^{t} d \eta \int_{\theta_{n+1}}^{\eta}\left|f\left(\xi, u_{n-1}(\xi), u_{n-1}^{\prime}(\xi), u_{n-1}^{\prime \prime}(\xi)\right) d \xi\right| \\
& \left.+\mid \int_{0}^{x} d t \int_{0}^{t} d \eta \int_{\theta_{n+1}}^{\theta_{n}} f(\xi), u_{n}^{\prime \prime}(\xi)\right)-f\left(\xi, u_{n-1}(\xi), u_{n-1}^{\prime}(\xi), u_{n-1}^{\prime \prime}(\xi)\right) d \xi \mid \\
\leq & \left.\int_{0}^{x} d t \int_{0}^{t} d \eta \int_{0}^{\eta} \mid f(\xi), u_{n-1}^{\prime \prime}(\xi)\right) \mid d \xi \\
& +M\left|\theta_{n+1}-\theta_{n}\right| \\
\leq & \left.M_{3} \int_{0}^{1} d t \int_{0}^{1} d \eta \int_{0}^{x} \mid u_{n}(\xi)-u_{n}^{\prime}(\xi), u_{n}^{\prime \prime}(\xi)\right)-f\left(\xi, u_{n-1}(\xi), u_{n-1}^{\prime}(\xi), u_{n-1}^{\prime \prime}(\xi)\right) \mid d \xi \\
& +\left|u_{n}^{\prime \prime}(\xi)-u_{n-1}^{\prime \prime}(\xi)\right| d \xi+\widetilde{M}_{n} \\
\leq & M_{3} \int_{0}^{x}\left|u_{n}(\xi)-u_{n-1}(\xi)\right|+\left|u_{n}^{\prime}(\xi)-u_{n-1}^{\prime}(\xi)\right|+\left|u_{n}^{\prime \prime}(\xi)-u_{n-1}^{\prime \prime}(\xi)\right| d \xi+\widetilde{M}_{n \prime}
\end{aligned}
$$

where $\widetilde{M}_{n}=M\left|\theta_{n+1}-\theta_{n}\right|$, we suppose $\left\{\theta_{n}\right\}$ is convergent, otherwise, we can take the convergent subsequence of $\left\{\theta_{n}\right\}$, thus $\widetilde{M}_{n} \rightarrow 0(n \rightarrow \infty)$. By doing this,

$$
\begin{aligned}
& \left|v_{n+1}^{\prime}(x)-v_{n}^{\prime}(x)\right| \\
& \quad \leq M_{3} \int_{0}^{x}\left|u_{n}(\xi)-u_{n-1}(\xi)\right|+\left|u_{n}^{\prime}(\xi)-u_{n-1}^{\prime}(\xi)\right|+\left|u_{n}^{\prime \prime}(\xi)-u_{n-1}^{\prime \prime}(\xi)\right| d \xi+\widetilde{M}_{n},
\end{aligned}
$$




$$
\begin{aligned}
& \left|v_{n+1}^{\prime \prime}(x)-v_{n}^{\prime \prime}(x)\right| \\
& \quad \leq M_{3} \int_{0}^{x}\left|u_{n}(\xi)-u_{n-1}(\xi)\right|+\left|u_{n}^{\prime}(\xi)-u_{n-1}^{\prime}(\xi)\right|+\left|u_{n}^{\prime \prime}(\xi)-u_{n-1}^{\prime \prime}(\xi)\right| d \xi+\widetilde{M}_{n} \\
& \left|v_{n+1}(x)-v_{n}(x)\right|+\left|v_{n+1}^{\prime}(x)-v_{n}^{\prime}(x)\right|+\left|v_{n+1}^{\prime \prime}(x)-v_{n(x)}^{\prime \prime}\right| \\
& \quad \leq 3 M_{3} \int_{0}^{x}\left|u_{n}(\xi)-u_{n-1}(\xi)\right|+\left|u_{n}^{\prime}(\xi)-u_{n-1}^{\prime}(\xi)\right|+\left|u_{n}^{\prime \prime}(\xi)-u_{n-1}^{\prime \prime}(\xi)\right| d \xi+3 \widetilde{M}_{n} \\
& \quad \leq 3 M_{3} \int_{0}^{x} 4 M_{1}\left(\left|v_{n}(\xi)-v_{n-1}(\xi)\right|+\left|v_{n}^{\prime}(\xi)-v_{n-1}^{\prime}(\xi)\right|+\left|v_{n}^{\prime \prime}(\xi)-v_{n-1}^{\prime \prime}(\xi)\right|\right)+4 \rho_{n} d \xi+3 \widetilde{M}_{n} \\
& \quad \leq \bar{M} \int_{0}^{x}\left|v_{n}(\xi)-v_{n-1}(\xi)\right|+\left|v_{n}^{\prime}(\xi)-v_{n-1}^{\prime}(\xi)\right|+\left|v_{n}^{\prime \prime}(\xi)-v_{n-1}^{\prime \prime}(\xi)\right| d \xi+\bar{\rho}_{n^{\prime}}
\end{aligned}
$$

where $\bar{M}=12 M_{1} M_{3}, \bar{\rho}_{n}=12 M_{3} \rho_{n}+3 \widetilde{M}_{n}, \lim _{n \rightarrow \infty} \bar{\rho}_{n}=0$.

When $n=1,\left|v_{2}(x)-v_{1}(x)\right|+\left|v_{2}^{\prime}(x)-v_{1}^{\prime}(x)\right|+\left|v_{2}^{\prime \prime}(x)-v_{1}^{\prime \prime}(x)\right| \leq \bar{M} x\left\|v_{1}-v_{0}\right\|_{C 2}+\bar{\rho}_{1}$.

When $n=2$,

$$
\begin{aligned}
\mid v_{3}(x) & -v_{2}(x)|+| v_{3}^{\prime}(x)-v_{2}^{\prime}(x)|+| v_{3}^{\prime \prime}(x)-v_{2}^{\prime \prime}(x) \mid \\
& \leq \bar{M} \int_{0}^{x}\left|v_{2}(\xi)-v_{1}(\xi)\right|+\left|v_{2}^{\prime}(\xi)-v_{1}^{\prime}(\xi)\right|+\left|v_{2}^{\prime \prime}(\xi)-v_{1}^{\prime \prime}(\xi)\right| d \xi+\bar{\rho}_{2} \\
& \leq \bar{M} \int_{0}^{x}\left(\bar{M} \xi\left\|v_{1}-v_{0}\right\|_{C^{2}}+\bar{\rho}_{1}\right) d \xi+\bar{\rho}_{2} \leq \frac{(x \bar{M})^{2}}{2}\left\|v_{1}-v_{0}\right\|_{C^{2}}+\bar{M} \bar{\rho}_{1} x+\bar{\rho}_{2} .
\end{aligned}
$$

Generally,

$$
\begin{aligned}
\left|v_{n+1}(x)-v_{n}(x)\right|+\left|v_{n+1}^{\prime}(x)-v_{n}^{\prime}(x)\right|+\left|v_{n+1}^{\prime \prime}(x)-v_{n}^{\prime \prime}(x)\right| \\
\leq \frac{(x \bar{M})^{n}}{n !}\left\|v_{1}-v_{0}\right\|_{C^{2}}+\sum_{k=0}^{n} \frac{(x \bar{M})^{k}}{k !} \bar{\rho}_{n-k} \\
\quad \leq \frac{(\bar{M})^{n}}{n !}\left\|v_{1}-v_{0}\right\|_{C^{2}}+\sum_{k=0}^{[n / 2]} \frac{(\bar{M})^{k}}{k !} \bar{\rho}_{n-k}+\sum_{k=[n / 2+1]}^{n} \frac{(\bar{M})^{k}}{k !} \bar{\rho}_{n-k} \\
\quad \leq \frac{(\bar{M})^{n}}{n !}\left\|v_{1}-v_{0}\right\|_{C^{2}}+\tilde{\rho}_{n} \sum_{k=0}^{[n / 2]} \frac{(\bar{M})^{k}}{k !}+\bar{\rho}_{k=[n / 2+1]}^{n} \frac{(\bar{M})^{k}}{k !} \longrightarrow 0, \text { as } n \longrightarrow \infty,
\end{aligned}
$$

where [.] denotes the integral part of ".", $\bar{\rho}=\max \left\{\bar{\rho}_{0}, \bar{\rho}_{1}, \ldots, \bar{\rho}_{n-[n / 2+1]}\right\}, \tilde{\rho}_{n}=$ $\max \left\{\bar{\rho}_{n}, \bar{\rho}_{n-1}, \ldots, \bar{\rho}_{n-[n / 2]}\right\}, \lim _{n \rightarrow \infty} \tilde{\rho}_{n}=0$. Thus, $\left\|v_{n+1}(x)-v_{n}(x)\right\|_{C^{2}} \rightarrow 0$, as $n \rightarrow \infty$, $x \in(\theta, 1]$. 
Let $\bar{v}_{n}(x)=v_{n}(1-x)$, then $\left\|\bar{v}_{n+1}(x)-\bar{v}_{n}(x)\right\|_{C^{2}} \rightarrow 0$, as $\left\|v_{n+1}(1-x)-v_{n}(1-x)\right\|_{C^{2}} \rightarrow$ $0, x \in(\theta, 1]$. Thus $\left\|v_{n+1}(x)-v_{n}(x)\right\|_{C^{2}} \rightarrow 0, x \in[0,1-\theta)$. Therefore, $\left|v_{n+1}^{\prime \prime}(0)-v_{n}^{\prime \prime}(0)\right| \rightarrow$ $0,\left|v_{n+1}(0)-v_{n}(0)\right| \rightarrow 0$, as $n \rightarrow \infty$.

After this transformation, the above result holds for (1.2). Next, we will give an equivalent representation of the solution of (1.2).

$$
\begin{aligned}
v_{n}(x)= & v_{n}(0)+\int_{0}^{x} d t \int_{0}^{t} d \eta \int_{0}^{\eta} f\left(\xi, u_{n-1}(\xi), u_{n-1}^{\prime}(\xi), u_{n-1}^{\prime \prime}(\xi)\right) d \xi \\
& -\frac{1}{2} x^{2} \int_{0}^{1} d \eta \int_{0}^{\eta} f\left(\xi, u_{n-1}(\xi), u_{n-1}^{\prime}(\xi), u_{n-1}^{\prime \prime}(\xi)\right) d \xi \\
u_{n}(x)= & P_{n} v_{n}, \quad n=1,2, \ldots
\end{aligned}
$$

Lemma 7.3. If $f(x, y, z, w)$ satisfies condition $H$, then $\left\|u_{n+1}-u_{n}\right\|_{C^{2}} \rightarrow 0$, as $n \rightarrow \infty$.

Proof. By $(7.11), v_{n}^{\prime \prime}(0)=-\int_{0}^{1} d \eta \int_{0}^{\eta} f\left(\xi, u n-1(\xi), u_{n-1}^{\prime}(\xi), u_{n-1}^{\prime \prime}(\xi)\right) d \xi$, then

$$
v_{n}(x)=\int_{0}^{x} d t \int_{0}^{t} d \eta \int_{0}^{\eta} f\left(\xi, u_{n-1}(\xi), u_{n-1}^{\prime}(\xi), u_{n-1}^{\prime \prime}(\xi)\right) d \xi+\frac{1}{2} x^{2} v_{n}^{\prime \prime}(0)+v_{n}(0) .
$$

From Lemma 7.2 and by using the same method, we can prove that $\left\|v_{n+1}(x)-v_{n}(x)\right\|_{C^{2}} \rightarrow 0, x \in[0,1]$. By Lemma 5.4 and $u_{n}=\alpha_{n-1} v_{n-1}$, it follows that $\left\|u_{n+1}-u_{n}\right\|_{C^{2}} \rightarrow 0$, as $n \rightarrow \infty$.

\section{Conclusion}

In this paper, we have shown the easier verification conditions for the existence of nonlinear third-order two-point boundary value problems. The method presented in this paper is based on the reproducing kernel space. We have presented reproducing kernel theorem for solving the nonlinear third-order two-point boundary value problems. Our results cannot be deduced trivially from any of the earlier published results and it is a pity that most of the people have not paid enough attention to the reproducing kernel theorem.

\section{Acknowledgments}

The research is supported by the Doctoral Initial Foundation of Harbin Normal University (KJB200817) and the Educational Department Scientific Technology Program of Heilongjiang Province (11541097).

\section{References}

[1] T. Y. Na, Computational Method in Engineering Boundary Value Problems, Academic Press, New York, NY, USA, 1979.

[2] N. S. Asaithambi, "A numerical method for the solution of the Falkner-Skan equation," Applied Mathematics and Computation, vol. 81, no. 2-3, pp. 259-264, 1997. 
[3] A. Asaithambi, "A finite-difference method for the Falkner-Skan equation," Applied Mathematics and Computation, vol. 92, no. 2-3, pp. 135-141, 1998.

[4] A. Asaithambi, "Numerical solution of the Falkner-Skan equation using piecewise linear functions," Applied Mathematics and Computation, vol. 159, no. 1, pp. 267-273, 2004.

[5] F. A. Howes, "Differential inequalities of higher order and the asymptotic solution of nonlinear boundary value problems," SIAM Journal on Mathematical Analysis, vol. 13, no. 1, pp. 61-80, 1982.

[6] S. Valarmathi and N. Ramanujam, "A computational method for solving boundary value problems for third-order singularly perturbed ordinary differential equations," Applied Mathematics and Computation, vol. 129, no. 2-3, pp. 345-373, 2002.

[7] M. R. Grossinho and F. M. Minhós, "Existence result for some third order separated boundary value problems," Nonlinear Analysis: Theory, Methods E Applications, vol. 47, no. 4, pp. 2407-2418, 2001.

[8] A. Cabada, M. R. Grossinho, and F. Minhós, "On the solvability of some discontinuous third order nonlinear differential equations with two point boundary conditions," Journal of Mathematical Analysis and Applications, vol. 285, no. 1, pp. 174-190, 2003.

[9] A. Cabada, M. R. Grossinho, and F. Minhós, "Extremal solutions for third-order nonlinear problems with upper and lower solutions in reversed order," Nonlinear Analysis: Theory, Methods E Applications, vol. 62, no. 6, pp. 1109-1121, 2005.

[10] M. Pei and S. K. Chang, "Existence and uniqueness of solutions for third-order nonlinear boundary value problems," Journal of Mathematical Analysis and Applications, vol. 327, no. 1, pp. 23-35, 2007.

[11] Z. Liu, L. Debnath, and S. M. Kang, "Existence of monotone positive solutions to a third order twopoint generalized right focal boundary value problem," Computers $\mathcal{E}$ Mathematics with Applications, vol. 55, no. 3, pp. 356-367, 2008.

[12] F. M. Minhós, "On some third order nonlinear boundary value problems: existence, location and multiplicity results," Journal of Mathematical Analysis and Applications, vol. 339, no. 2, pp. 1342-1353, 2008.

[13] Y. Feng and S. Liu, "Solvability of a third-order two-point boundary value problem," Applied Mathematics Letters, vol. 18, no. 9, pp. 1034-1040, 2005.

[14] M. Cui and Z. Deng, "Solutions to the definite solutions problem of differential equations in space $W_{2}^{l}, "$ Advances in Mathematics, vol. 17, pp. 327-329, 1988.

[15] C.-1. Li and M.-G. Cui, "The exact solution for solving a class nonlinear operator equations in the reproducing kernel space," Applied Mathematics and Computation, vol. 143, no. 2-3, pp. 393-399, 2003.

[16] M. Cui and Y. Lin, Nonlinear Numerical Analysis in the Reproducing Kernel Space, Nova Science, New York, NY, USA, 2009. 